\title{
The Accuracy of Multidetector Row CT Angiography in the Evaluation of Living Kidney Donors
}

\author{
Seksan Chitwiset MD ${ }^{1}$ \\ Vorapot Choonhaklai ${ }^{2}$ \\ ${ }^{1}$ Department of Radiology, Rajavithi Hospital, College of Medicine, Rangsit University, Bangkok, Thailand. \\ ${ }^{2}$ Division of Urology, Department of Surgery, Rajavithi Hospital, College of Medicine, Rangsit University, Bangkok, Thailand.
}

\begin{abstract}
Purpose: To assess the value of MDCT in evaluation of the anatomy of living kidney donors and in revealing the prevalence of renal vascular variations.

Materials and Methods: This retrospective study was approved by the Ethics committee of Rajavithi hospital(NO.172/2555). The accuracy of the images of vessel structures obtained by using 64-row multidetector computed tomography (MDCT) angiography in comparison with the actual vessel structures observed during surgery was analyzed retrospectively.

We analyzed 20 patients who had undergone donor nephrectomy from 2008 to 2012 . Preoperative MDCT angiography was performed, and the surgeons themselves subsequently recorded the operative findings. Results: Nephrectomy was performed on the left side in all 20 patients. The accuracy levels of MDCT for the artery and the vein were $90 \%$ and $95 \%$ respectively.

Conclusions: MDCT angiography is a good investigative technique for the preoperative evaluation of the anatomy of vessels.

Key Words: Kidney transplantation; Living donors; Renal artery; Renal veins; MDCT
\end{abstract}

Correspondence to:

Jitwiset S. Diagnostic Radiology, Radiologic department,Rajavithe Gospital, Collecge of Medicine, Rangsit University, 2 Phyathai Road, Ratchathewi, Bangkok 10400,Thailand

Phone:0-2354-8180 ext 3025, Fax:0-2354-8093

e-mail: seksanchit@hotmail.com 


\section{Introduction}

End-stage renal failure is a growing health problem in Thailand. The number of sufferers is increasing due to the prevalence of many underlying diseases and prolonged life expectancy. Patients who suffer from this problem end up having to survive with hemodialysis or continuous ambulatory peritoneal dialysis (CAPD). Renal transplantation is a good treatment choice because it offers improved quality of life and is cost-effective. Cadaveric kidneys available for transplantation are limited: the number of patients waiting for cadaveric kidney transplantation is increasing, and few patients are able to have such transplants because of the lack of availability of cadaveric organs. To make up for this shortage, live kidney donation is now a widely accepted practice, with better recipient tolerance and better renal graft survival than can be achieved with cadaveric donor transplantation $^{1-3}$.

Preoperative imaging work-up of living renal donors involves anatomic and functional evaluation of donor kidneys for the selection of a suitable donor and for the planning of surgery. In donor nephrectomy, it is important to understand the exact anatomy of the blood vessels. Information about the number of renal arteries and early division of the main renal artery is important when deciding whether to remove the right or left kidney. This information is also important for minimally-invasive surgery in order to decrease blood loss and the length of hospital stay by reducing complications and accelerating recovery ${ }^{4.5}$.

In the past, preoperative evaluation were included intravenous pyelography (IVP), angiography, and abdominal sonography ${ }^{6,7}$. With the introduction of multidetector computed tomography (MDCT), those methods have been replaced by computed tomography (CT) angiography ${ }^{8}$. CT angiography is less invasive than conventional angiography and has the advantage of allowing not only highly accurate assessment of the main vessel (renal vein, renal artery) and ureter structure, but also detection of renal cystic diseases, renal parenchymal lesions, tiny stones, and hilar vessels ${ }^{9-11}$.

Many previous studies have been carried out to examine the accuracy of CT angiography in determining vessel structures. The level of accuracy for the main vessels was reported to vary from $90 \%$ to $100 \%$, and for the minor vessels from $75 \%$ to $100 \%{ }^{12-15}$. One reason for the variation in accuracy may be from different $\mathrm{CT}$ protocols.

The objective of this study was to assess the accuracy of CTA images for pre-operative evaluation of renal vascular anatomy in comparison with the actual vessel structure observed during surgery.

\section{Materials and Methods}

This retrospective study was approved by the Ethics committee of Rajavithi hospital (NO.172/2555). Between 2008 and 2012, the number of kidney donors who were evaluated with MDCT in our center (Rajavithi Hospital) were 8 males and 12 females (a total of 20 donors) whose ages ranged from 20-53 years old (mean age $=40.6$ years old). All 20 donors underwent nephrectomy

\section{CT protocols}

All CT examinations were performed with a standardized examination protocol using a multislice 64 detector-row helical CT scanner (Lightspeed VCT, GE Medical Systems, Milwaukee, USA). Scanning was initiated with a scout image covering the whole abdomen. Precontrast imaging was performed with 
$5 \mathrm{~mm}$ slices and the table rotation time was $0.6 \mathrm{sec}$ with $120 \mathrm{kV}$ and automated calculation of mAs. After a plain scan of the abdomen (scout film) had been taken, an 18-20 gauge intravenous catheter was placed in the antecubital vein. Using an automatic injector, $100 \mathrm{ml}$ of the contrast material was injected at a flow rate of $4 \mathrm{ml} / \mathrm{sec}$. The arterial phase was obtained after 12 seconds, and the venous phase 60 seconds after the initiation of the contrast bolus. The arterial phase included a volume covering the diaphragm to the pelvis. After acquiring the images, arterial phase and venous phase images were reconstructed by axial $3 \mathrm{~mm}$ and $1 \mathrm{~mm}$ images and coronal $3 \mathrm{~mm}$ images.

The reconstructed images were processed with a commercially available work station (ADW 4.3: GE medical systems). Two- and three-dimensional maps of the renal arteries, renal veins and ureters were generated by using maximum-intensity projection (MIP), volume rendering techniques and multiplanar reformation. In addition, a 3D reconstruction image was acquired with $0.625 \mathrm{~mm}$ slice thickness. All CT angiographies were interpreted by a radiologist before donor nephrectomy. During the evaluation, the main vessel structures of the right and left sides were described, together with the path of the hilar veins. Early branching renal artery exists when the first branch of the renal artery originates within 2.0 $\mathrm{cm}$ from the lateral wall of the aorta.

\section{Results}

The radiological findings of the $\mathrm{CT}$ of the main vessels were compared with the surgical findings of the 20 donors (8 males and 12 females. All nephrectomies were performed on the left side with open flank nephrectomy in 18 donors and with laparoscopic approach in the other 2. The mean age of the patients was 40.6 years old (range, 20-53 years old). CT showed that a small simple renal cyst (diameter less than $1 \mathrm{~cm}$ ) was present in one of 20 donor kidneys. None of the selected donor kidneys was noted to have renal stones, fibromuscular dysplasia of renal artery, or any congenital vascular abnormality on multidetector CT images.

\section{Renal artery}

A total of 23 renal arteries in the 20 donor kidneys were depicted with CT. Only 3 kidneys were shown to have more than one renal artery (2 renal arteries in each kidney). During surgery, 23 arteries were also identified in the 20 kidneys, 17 of the 20 kidneys (85\%) had a single artery and 3 of the 20 kidneys (15\%) had more than one artery. One accessory artery, the superior polar artery, in 1 kidney was initially missed on CT. The sensitivity and specificity of CTA for detection of variant anatomy of renal arteries were $66.66 \%$ and $94.11 \%$ respectively. The accuracy of CT in detection of renal arteries, positive predictive values, and negative predictive values were $90 \%, 66.67 \%$ and $94.11 \%$ respectively, indicating good agreement with surgical findings. The one accessory renal artery that had been missed at the CT initial interpretation was identified in a retrospective review.

\section{Renal vein}

A total of 20 renal veins were identified in CT images of 20 donor kidneys. During surgery, 21 renal veins were identified in 20 kidneys; one kidney had 2 renal veins, and each of the remainder had just one renal vein. The sensitivity and specificity of CT for the identification of variant anatomy of renal veins were $50 \%$ and $100 \%$ respectively. In surgery, the overall CT findings were concordant in 19 of the 


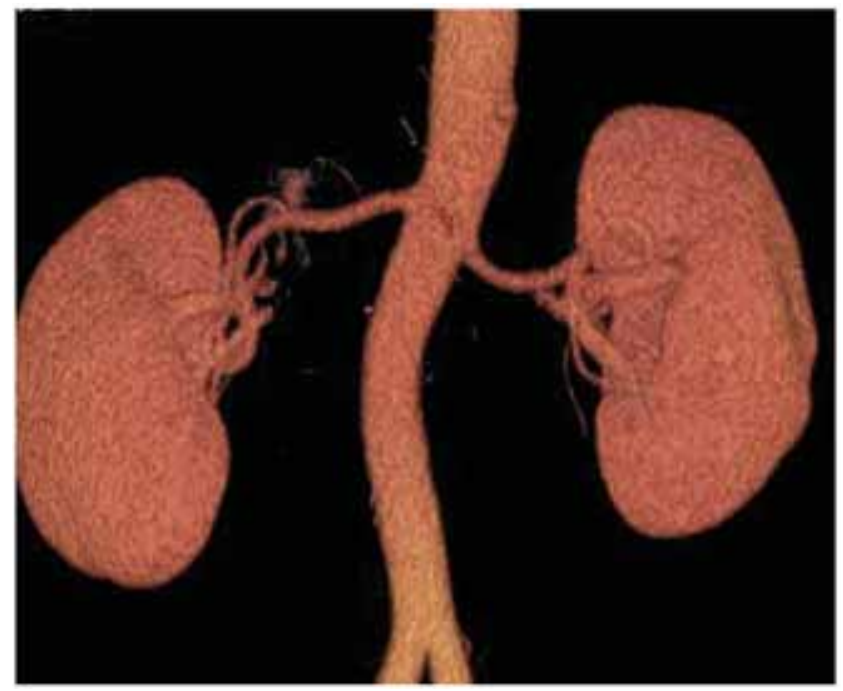

Fig. 1 A 3-D volume-rendered (VR) image of a 48-year-old female renal donor obtained with a 64-slice MDCT demonstrating single right and left renal artery

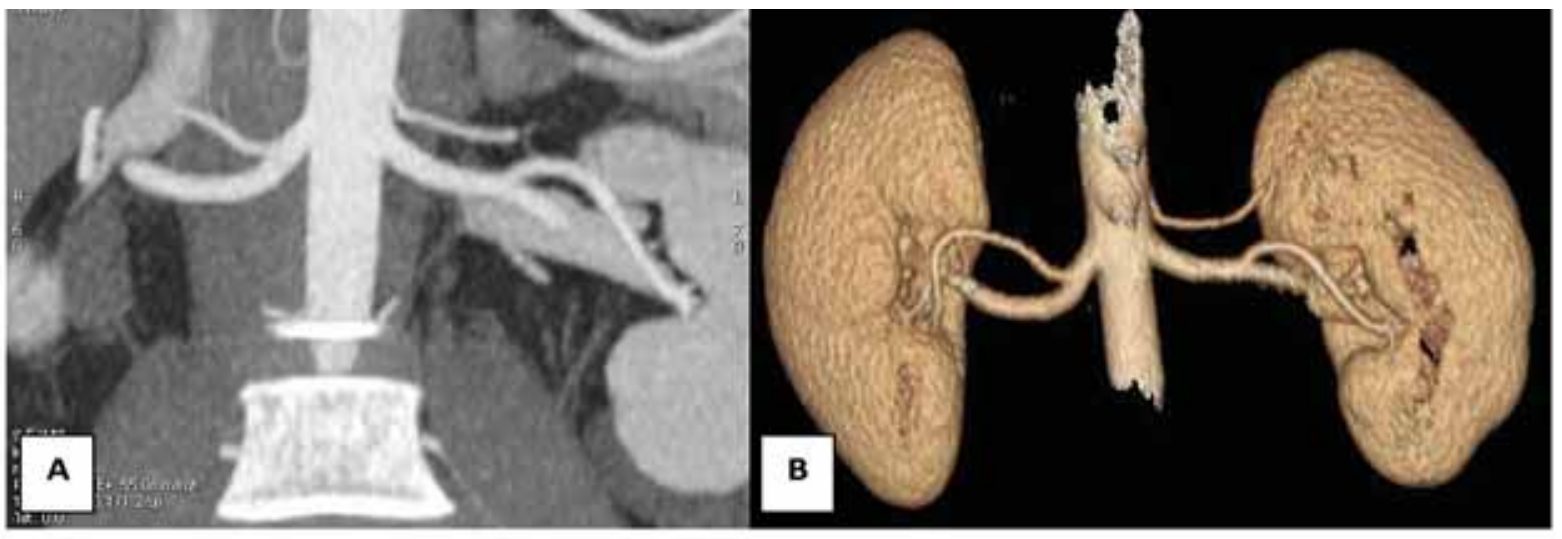

Fig. 2 (A) A 3-D maximum intensity projection(MIP) image and (B)a 3-D volume-rendered (VR) of a 20-year-old female renal donor obtained with 64-slice CT show two left renal arteries and early branching of single right renal artery $(7 \mathrm{~mm}$. from aorta) 


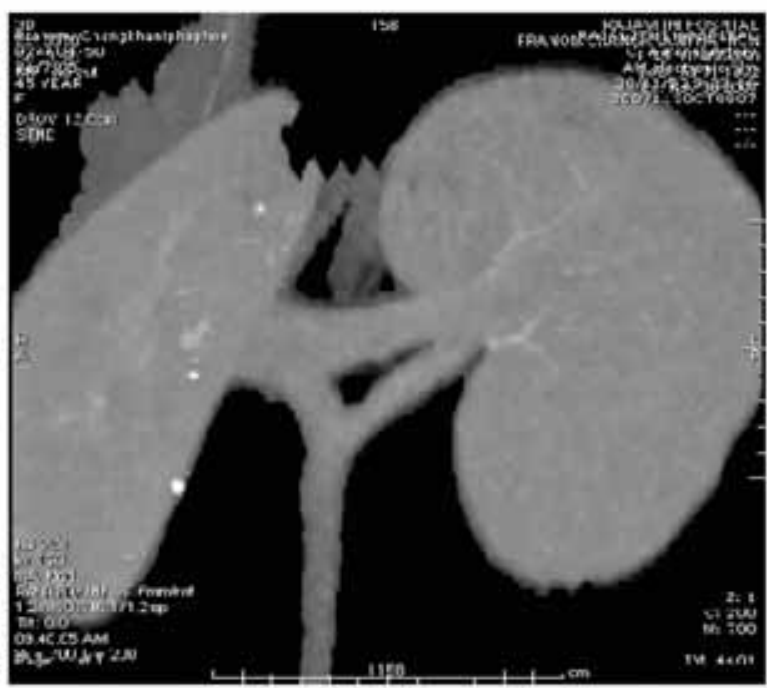

Fig. 3 A 3-D maximum intensity projection (MIP) image of a 45-year-old female renal donor obtained with 64-slice MDCT demonstrating double left renal vein with left gonadal vein drain into the inferior left renal vein

20 kidneys (accuracy 95\%). The positive predictive and negative predictive values were $100 \%$ and $94.73 \%$ respectively.

\section{Discussion}

Renal transplantation is a good therapy for patients with end-stage renal disease. Due to the limited number of cadaveric organs available, patients are having to suffer for extended periods of time before undergoing cadaveric kidney transplantation; consequently, the number of living donors has been increasing in recent times ${ }^{3,12}$. It is crucial to evaluate the donor's vascular anatomy before the removal of a kidney with any surgical technique, especially when using a laparoscopic approach because of the limited exposure during the procedure ${ }^{13,14}$. Understanding the vascular anatomy of the kidney is an essential part of pre-operative evaluation of living kidney donors, and it helps the clini- cians to choose suitable donors and decide whether the left or the right kidney should be used ${ }^{15}$. The anatomic information required before conventional open and minimally-invasive surgery in living kidney donors includes number, length, location and branching patterns of the renal arteries, as well as the status of the donor kidneys and their venous and blood collecting systems ${ }^{8,16}$.

In the past, conventional angiography was used to evaluate renal arteries before surgery ${ }^{10,17,18}$. It was a rather invasive procedure and donors had to stay in hospital for a few days after the angiographic procedure with the risk of endothelial damage.

Several investigators have used single-detector row helical CT for predicting renal vascular anatomy $y^{9,14}$ and some have reported good correlation with both catheter angiography and surgery $^{5,15-18}$.

Improvements in CT technology have enabled 
multidetector row CT to achieve increased speed of scanning and enhanced spatial resolution with advances in post processing methods ${ }^{19}$. Multidetector row CT provides greater volume coverage with superior quality three-dimensional angiograms. Delayed CT urographic imaging of excretory phase CT is helpful in evaluating pyelocalyceal and ureter anatomy and obviates the need for intravenous pyelography.

The aim of this study was to evaluate the accuracy of renal CT angiography obtained by the use of 64-slice MDCT for prediction of the vascular anatomy and its variations in living kidney donors. It can be concluded that, for renal arteries, CT findings were concordant with those from surgery in 18 of the 20 donors (accuracy 90\%). Although one accessory artery of about $1.5 \mathrm{~mm}$ in diameter had been missed in the initial CT interpretation, it was identified on transverse CT scan alone in a retrospective review of CT data.

Accurate identification of renal veins was achieved in 19 of the 20 donor kidneys (accuracy $95 \%)$. The initially-missed accessory renal vein in CT was seen in retrospective review.

The accuracy of the renal vascular anatomy was reported to be high, at $90-100 \%$, and in studies of multidetector row CT, successful identification of minor venous anomalies was reported to be $75-$ $100 \%$. The results of this study correspond well with those of earlier studies with high sensitivity and accuracy in the assessment of renal vasculature ${ }^{11,21-25}$.

There have been previous reports about vascular variants in living Thai donors, in which supernumerary renal arteries were found in about 18.5$27 \%$ of kidneys. The variations that were found on 64-row MDCT were accessory renal artery $=26.46 \%$; early branching $=0.62-22.4 \%$; and venous variant $=1.85 \%{ }^{11,26}$. Accessory renal arteries were found in about $15 \%$ of kidneys in this study.

Preoperative evaluation is important, especially for laparoscopic surgery because of its limited field of view. Conversion to the open approach is not infrequent, the most common reason being vascular injury ${ }^{27,28}$. Therefore, special attention must be paid to the assessment of donor renal vessels and collecting system for selection of suitable kidneys and for planning surgery.

There were some limitations in our study. First the number of donors was low, resulting in a low number of variations. Second, this study was a retrospective one, so many donors were excluded from this study due to incomplete information; furthermore, the study reviewed cases involving many radiologists with varying experience, and it is important to note that we chose to select kidneys with normal anatomy and fewer anomalies for donor nephrectomy. Therefore, the performance of multidetector row CT in the evaluation of more complex vascular and excretory anatomy and anomalies could not be compared.

\section{Conclusion}

MDCT angiography can provide highly accurate assessment of renal vascular anatomy in living kidney donors that will be helpful for preoperative planning.

\section{Acknowledgement.}

The present study was support by Rajavithi Hospital Research fund.

\section{References}

1. Poli F, Scalamogna, CariloM, Porta E, Sirchia G. An algo- 
rithm for cadaver kidney allocation base on a multivariate kidney graft survival and function. Transpl Int 2000; 13(supple 1):S259-62

2. Goel C, Modlin Cs, Mottoo AM, Derweesh IH, Flechner SM, Streem S, et al. Fate of donor kidney:laparoscopic versus open technique. J Urol 2004;172(6 pt 1):2326-30.

3. Annual Report of the U.S Scientific Registry of Transplant Recipients and the Organ Procurement and Trasplantation Network-Transplant Data:1988-1994. UNOS, Richmond,VA and the Division of Transplantation, Bureau of Health Resources and Services Administration,U.S. Department of Health and Human Services, Rockville, MD, 1995

4. Giessing M. Laparoscopic Living-donor nephrectomy. Nephrol Dial Transplant 2004;19(supple4):iv36-40.

5. Rydberg J, Kopecky KK, Tann M, Persohn SA, Leapman SB, Filo RS, et al. Evaluation of preoperative of prospective living renal donor for laparoscopic nephrectomy with multisection CT:the marriage of minimally invasive imaging with minimal invasive surgery. Radiographics 2001;21 Spec No:S223-6.

6. Derauf B, Goldberg ME. Angiographic assessment of potential renal transplant donors. Radiol Clin North Am 1987;25:261-5.

7. Shaffer D, Sahyoun AI, Madras PN, Monaco AP. Two hundred and one consecutive living-donor nephrectomies. Arch Surg 1998;133:426-31.

8. Cochran ST, Krasny RM, Danovitch GM, Rajfer J, Barbaric ZM, Wilkinson A, et al. Helical CT angiography for examination of living renal donors. AJR Am J Roentgenol 1997;168:1569-73

9. Kawamoto S, Montgomery RA, Lalwer LP, Horton KM, Fishman EK. Multidetector CT angiography for preoperative evaluation of living laparoscopic kidney donors. AJR Am J Roentgenol 2003;180:1633-8.

10. Platt JF, Ellis JH, Korobkin M, Reie K. Helical CT evaluation of potential kidney donors:finding in 154 Subjects. AJR 1997;169:1325-30

11. Raman SS, Pojchmarnwiputh S, Muangsomboon K, SchuIam PG, Gritsch HA, Lu DS. Utility of 16-MDCT angiography for comprehensive preoperative evaluation of laparoscopic renal donor. AJR 2006;186:1630-8.

12. Cecka JK, The OPTN/UNOS renal transplant registry, Clin Transpl 2005;1-16.
13. Ratner LE,Ciseck IJ, Moore RG, Cigarroa FG, Kaufman HS,Kavoussi LR. Laparoscopic live donor nephrectomy. Transplantation 1995;60:1047-9.

14. Rubin GD, Alfrey EJ, Dake MD, Semba CP, Sommer FG, Kuo PC, et al. Assessment of living donors with spiral CT. Radiology 1995;195:457-62.

15. Satyapal KS, Haffejee AA, Singh B, Ramasaroop L, Robbs JV, Kalideen JM. Additional renal arteries: incidence or morphometry, Surg Radiol Anat 2001;23:33-8.

16. Del Pizzo JJ, Sklar GN, You-Cheong JW, Levin B, Krebs T, Jacobs SC. Helical computerized tomography arteriography for evaluation of live related donor undergoing laparoscopic nephrectomy. J Urol 1999;162:31-4.

17. Burgos FJ, Pascual J, Mareen R, Garcia-Navas R, Gomez $\mathrm{V}$,Ortuno J. The role of imaging techniques in renal transplantation. World J Urol 2004;22(5):399-404.

18. Leim YS, Kock MC, ljzermans JN, Weimar W, Visser K, Hunink MG. Living renal donors:optimizing the imaging strategy-decision and cost-effectiveness analysis. Radiology 2003;180(6):53-62

19. Rydberg J, Laiang Y,Teague SD. Fundamentals of multichannel CT. Radiol Clin North Am 2003:41:465-74.

20. Holden A, Smith A, Dukes P, Pilmore H, Yasutomi M. Assessment of 100 Live Potential Renal Donors for laparoscopic nephrectomy with multidetector row helical CT. Radiology 2005;237:973-80.

21. Sahani DV, Rastogi N, Greenfield AC, Kalva SP, Ko D, Saini S, Multidetector row CT in evaluation of 94 living renal donors by readers with varied experience, Radiology 2005;235:905-10.

22. Raman SS, Pojchamarnwiputh S, Muangsomboon K, Schulam PG, Gritsch HA, Lu DS. Utility of 16-MDCT angiography for comprehensive preoperative vascular evaluation of Laparoscopic renal donors, AJR 2006;186: 1630-8.

23. Apisarnthanarak $P$, Suvannarerg V, Muangsomboon K, Taweemonkongsap T, Hargrove NS. Renal vascular variants in living related renal donors: evaluation with CT angiography, J. Med Assoc Thai. 2012;95(7):941-8.

24. Kim JK, Park SY, Kim HJ, Kim CS, Ahn HJ, Ahn TY, et al. Living donor kidneys:usefulness of multidetector row CT for comprehensive evaluation. Radiology 2003;229: 869-76. 
January-April 2013, Volume XIX No.I

25. Namasivayam S, Small WC, Kalra MK,Torres WE, Newell KA, Mlttal PK. Multidetector-row CT angiography for preoperative evaluation of potential laparoscopicrenal donors:how accurate are we? Clin imaging. 2006;30: 120-6.

26. Ekkasit S, Chutcharn K,Supakajeee S. Anatomical Cariation of Renal Vessels by 64 Slices Multidetector Computed Tomography(MDCT) Scan of abdomen at Pramongkutklao Hospital.RTA Med J;664:75-83.
27. Rydberg J, Kopecky KK, Tann M, Persohn SA, Leapman $\mathrm{SB}$, Filo RS, et al. Evaluation of prospective living renal donors for laparoscopic nephrectomy with multisection $\mathrm{CT}$;the marriage of minimally invasive imaging with minimally invasive surgery. Radiographics 2001;21:223-36.

28. Richstone L, Seideman C, Baldinger L, Permpandkosol SS, Jarrett TW, Su LM, et al. Conversion during laparoscopic surgery:frequency,indication and risk factors. J Urol 2008;80:855-9. 